\title{
A Role of ICH- GCP in Clinical Trial Conduct
}

\author{
Pranali Wandile* and Ravindra Ghooi \\ Clinical Research Administration at Kearney Regional Medical Center, Kearney, Nebraska, 68845, USA
}

*Corresponding author: Pranali Wandile, Clinical Research Administration at Kearney Regional Medical Center, Kearney, Nebraska, 68845, USA, Tel: 7346450153; Email: pranali_wandile2006@yahoo.com

Received date: January 05, 2017; Accepted date: February 13, 2017; Published date: February 23, 2017

Copyright: $\odot 2017$ Wandile P, et al. This is an open-access article distributed under the terms of the Creative Commons Attribution License, which permits unrestricted use, distribution, and reproduction in any medium, provided the original author and source are credited.

\begin{abstract}
The Good Clinical Practice Guideline of the International Conference on Harmonization (ICH-GCP) is an international ethical, scientific and quality standard to harmonize technical procedures and standards, improve quality, speed time to market the drug. This standard is for designing, conducting, performing, monitoring, auditing, recording, analyzing and reporting clinical trials involving human subjects.

Mushrooming regulatory requirements of different countries made new drug research very expensive and time consuming, simply because different countries had different requirements. The ICH standardized the requirements so that a drug developed as per the GCP guidelines could be acceptable to any member country of the ICH. Past history of human research abuses led to the inclusion of ethical standards to make them uniform across the $\mathrm{ICH}$ region. The guideline lays emphasis on protecting rights, safety, and welfare of human study subjects who participate in studies. In last two decades, these standards have evolved and become pillars of successful global drug development, which we have today. These standards have been revised and updated in 2016, but the core principles remain the same.
\end{abstract}

\section{Introduction}

\section{Role of ICH- GCP in clinical trial conduct}

The International Council on Harmonization's Technical Requirements for Registration of Pharmaceuticals for Human Use (ICH) is a project that brings together regulatory authorities of Europe, Japan, United States and experts from the pharmaceutical industry in the three regions to discuss scientific and technical aspects of pharmaceutical product registration [1].

Good Clinical Practice (GCP) is an international ethical and scientific quality standard for designing, conducting, performing, monitoring, auditing, recording, analyzing and reporting trials that involve the participation of human subjects [2]. The objective of this guideline is to provide a unified standard for the region to facilitate the mutual acceptance of clinical data by the regulatory authorities in these regions.

Accepting and following these ethical standards provides an assurance of protection of rights, safety, well-being of research study subjects. These standard guidelines follow the principles that originated in Nuremberg code and Declaration of Helsinki and lead to the generation of credible clinical trial data [3].

\section{History of ethical guidelines}

The foundations for the conduct of clinical research are ethical guidelines given below

\footnotetext{
- The Nuremberg Code

- The Declaration of Helsinki

- The Belmont Report
}

- International Conference on Harmonization (ICH-GCP)

- Code of Federal Regulations

Historical events have been responsible for the development of these codes and guidelines, which have been based on single philosophy that has been voiced in Belmont Report. These codes and guidelines have formed the basis of the Good Clinical Practice of $\mathrm{ICH}$, which borrows freely from various codes and guidelines to form unified code of practice [4].

\section{7-Nuremberg code}

At the end of the World War II the Allied Forces came across evidence that Nazis had conducted a large number of experiments in the name of research. These experiments were conducted on civil or war prisoners, and often on Jews who were considered as sub-human by the Nazis. The scientific rationale of these experiments was often flawed and most of them ended in extreme physical and mental hardship for the participants. A very large number of participants died in experiments, in which they were forced to participate. A total of 23 officials, doctors and administrators were prosecuted for crimes against humanity at the Palace of Justice in Nuremberg, in what have been known as Doctors' Trial.

Most of the accused were indicted for the crime of performing brutal experiments on human subjects under the name of medical science. Some of the accused doctors claimed that there was no law in place, which defined what experiments on human subjects were permissible and in the absence of such a law no crime could be attributed to them. Here it may be mentioned that they overlooked the Berlin Code of 1900, and the Guidelines for Human Experimentation of 1931, both of which had been developed in their own country. Undeterred by the absence of any law, seven doctors were handed 


\section{(10.4172/2155-9627.1000297}

Page 2 of 5

down death sentences, seven were acquitted and the rest handed down sentences ranging from 5 to 25 years of imprisonment, by the court.

Following the Doctors' Trial at Nuremberg, the medical experts Drs. Leo Alexander, Andrew Ivy and Harold Seibling prepared a list of conditions under which medical experiments could be permitted [5]. This list was subsequently published as the Nuremberg Code and included ten ethical principles to protect the rights and wellbeing of study subjects [6].

The ten point code so formulated contains the following:

1. Participation in studies is voluntary and after the participant provides an Informed consent.

2. The experiment should be useful to mankind.

3. Human experiments should be based on results of prior animal experiments.

4. Physical and mental suffering to subjects should be avoided.

5. No experiment that may lead to death or disability to subject should be undertaken.

6. The risk should not exceed the humanitarian importance of the problem to be solved.

7. Human subjects should be protected against even remote possibilities of harm.

\section{Only qualified scientists should conduct medical research.}

9. Human subjects should be free to end an experiment at any time.

10. The scientist in charge must be prepared to end an experiment at any stage [4].

The Nuremberg code does not have any legal status and is not enforceable in any part of the world, however it has formed the foundation on which later codes have been based [7]. It is unfortunate that a number of points in the Nuremberg code have been copied from an earlier guideline for research on human subjects promulgated by the Weimar Government in 1931 [8].

\section{2- Kefauver-Harris Amendment}

In 1962 after thalidomide tragedy, Kefauver-Harris Amendment was passed and it is responsible for current IND (Investigational New Drug) Application regulations. The following were changes brought about by this amendment in the drug law.

a) The voluntary consent of all subjects in non-therapeutic and therapeutic research became mandatory.

b) Drugs were required to have proven efficacy and safety.

c) An FDA approval was required for the marketing of a new drug, (not just the review and 60-day waiting time).

d) Reporting of adverse events became mandatory.

e) All the NDAs approved between 1938-1962 were to be reviewed by FDA, to check if products still met the new efficacy and safety standards [9].

\section{4-Declaration of Helsinki}

The absence of a legal force behind the Nuremberg Code made it a toothless tiger. While widely respected, no country in the world followed the code. Widespread ethical violations continued throughout the world, hence the World Medical Association adopted Declaration of Helsinki in their annual meeting in 1964. It included a statement binding the physicians to the welfare of their patients stating, "The health of my patient will be my first consideration".

In the last 52-years, the Declaration has been revised many times, major revisions have been made seven times (the most recent at the General Assembly in October 2013 at Fortaleza in Brazil). It is very important document in medical research history and is the basis of GCP and most subsequent ethics guidelines issued worldwide. It reiterated the need for written consent, and introduced the concept of prior review and approval of protocol by IRB [10]. Additionally, it introduced newer concepts like post-trial access, marked reduction in the use of placebo among others. It is unfortunate that the United States officially does not accept the Declaration of Helsinki [11], this leads to situations where trials in United States run foul of guidelines of some countries [12].

Principles-Well-being of subject is a priority in research, this includes respect for persons, protection of subjects' rights and safety and special protection for vulnerable population. It included most of the principles from Nuremberg Code, and added a number of new ones. Each revision has made the Declaration more comprehensive and inclusive.

\section{9-The Belmont Report}

In 1974 Congress passed the National Research Act and created National Commission for the Protection of Human Subjects of Biomedical and Behavioral Research. This commission prepared Belmont Report in 1979. Incidentally, the report is named after the room in the Smithsonian Institute where the National Research Commission held its meetings. The report covered medical ethics in three basic ethical principles for the protection of human research subjects. Principles were-respect for person, beneficence and justice. Incidentally the fourth principle of non-maleficence was added as an afterthought and is not actually a part of the report [13].

Respect-Respect to persons means giving them the autonomy to decide what may be done to them. The most powerful instrument to achieve autonomy is the informed consent; a process that gives the subject the right to refuse to participate in research and assures the protection of subject's safety, privacy, confidentiality, while extending special protection to vulnerable subjects.

- Beneficence: This principle requires researchers to maximize research benefits and minimize associated harms. Research-related risks must be weighed against expected benefits and found commensurate with the expected benefits.

- Justice: Include equitable selection and fair treatment of research subjects and equitable distribution of benefits and risks of research.

The Belmont report has never been reviewed or revised. In fact the nature of the document is such that any changes have not been necessary. Unlike the Declaration of Helsinki, which speaks of the nuts and bolts or actions that need to be taken, the Belmont report speaks of the principles of ethics. The basic principles of ethics are also basic to all human relations, and have not changed so far, nor are they expected to change [14]. 


\section{History and initiation of ICH}

In many countries, before the birth of ICH-GCP, there was a rise in drug development guidelines, laws, and regulations due to safety, efficacy and quality. The pharmaceutical industry all over was looking to capture global markets, but due to the differences in technical requirements in different countries, industry needed to duplicate their work before submission of an IND or NDA in a different country. This process led to duplication of efforts, increased research and developmental cost, increase in healthcare cost and delay in marketing drugs. Patients in urgent need of drugs had to wait for long, and often drugs did not become available during their lifetime. All these concerns compelled leading countries involved in drug discovery to rationalize and harmonize regulations among themselves [15].

In 1980 European Community (current European union) achieved success in development of single pharmaceutical market in Europe, which proved that harmonization was possible.

In April 1990 industry associations and regulatory agencies of Europe, Japan and the US met in Brussels, leading to inception of ICH. It was decided to develop harmonization guidelines in sections such as safety, quality and efficacy, with a miscellaneous section [16]. These would eventually become the basis for developing, testing and authorizing new medicinal products [17].

The participants in the development of these guidelines were representatives of regulators and pharmaceutical companies from EU, Japan, United States, while Australia, Canada, Nordic countries and WHO were observers [18].

The Guideline "E6" which was approved on 17 July 1996 and implemented from 17 January 1997 became the most important guideline that covered clinical studies. Commonly known as the $\mathrm{ICH}$ GCP guideline, its objective was to provide a joint standard for the $\mathrm{ICH}$ region for mutual acceptance of clinical data by regulatory authorities. This guidance was to be followed when generating clinical trial data that are intended to be submitted to regulatory authorities in the ICH region [19].

The purpose of ICH is to reduce or eliminate the need to duplicate the testing carried out during new drug development process, by suggesting the ways to achieve greater harmonization in the technical requirements for product registration. Harmonization would lead to a more economical use of human, non-human, material resources, reduce unnecessary delay in development and improve availability of new medicines globally, while maintaining safeguards on quality, safety, and efficacy, regulatory obligations to protect the people.

Harmonization of regulations between different countries, having different first languages presents hitherto unexpected problems. The first among which is the language; persons in one region must perfectly what another in a different means. The meaning of words changes as one crosses different latitudes, and it therefore became necessary to have standard languages (ICH adopted only English and French) and every word to have a standard meaning understood by all. For this reason they had to create a glossary which is in fact the first chapter of the Guideline E6.

Currently only EU, US and Japan follow ICH guidelines, however since the ICH region holds over $70 \%$ of the pharmaceutical market, most other countries that are involved in drug development and hope to market their products in the ICH region, also follow them. Many of these countries (including India) have modified their national guidelines to make them at par with the ICH guidelines. It is true that many countries are not following these guidelines, but these countries are not involved in drug development.

There are 13 core principles of ICH-GCP and they are as follows [2]:

1. Clinical trials should be conducted in accordance with the ethical principles that have their origin in the Declaration of Helsinki, and that are consistent with GCP and the applicable regulatory requirement(s).

2. Before a trial is initiated, foreseeable risks and inconveniences should be weighed against the anticipated benefit for the individual trial subject and society. A trial should be initiated and continued only if the anticipated benefits justify the risks.

3. The rights, safety, and well-being of the trial subjects are the most important considerations and should prevail over interests of science and society.

4. The available nonclinical and clinical information on an investigational product should be adequate to support the proposed clinical trial.

5. Clinical trials should be scientifically sound, and described in a clear, detailed protocol.

6. A trial should be conducted in compliance with the protocol that has received prior institutional review board (IRB)/independent ethics committee (IEC) approval/favorable opinion.

7. The medical care given to, and medical decisions made on behalf of, subjects should always be the responsibility of a qualified physician or, when appropriate, of a qualified dentist.

8. Each individual involved in conducting a trial should be qualified by education, training, and experience to perform his or her respective $\operatorname{task}(\mathrm{s})$.

9. Freely given informed consent should be obtained from every subject prior to clinical trial participation.

10. All clinical trial information should be recorded, handled, and stored in a way that allows its accurate reporting, interpretation, and verification.

11. The confidentiality of records that could identify subjects should be protected, respecting the privacy and confidentiality rules in accordance with the applicable regulatory requirement(s).

12. Investigational products should be manufactured, handled, and stored in accordance with applicable good manufacturing practice (GMP). They should be used in accordance with the approved protocol.

13. Systems with procedures that assure the quality of every aspect of the trial should be implemented.

The FDA endorsed and implemented GCP by accommodating the guidelines in following federal regulations.

- 21 CFR 11- Electronic Records \& Signatures

- 21 CFR 50- Protection of Human Subjects In Clinical Trials

- 21 CFR 54- Financial Disclosure

- 21 CFR 56- Institutional Review Boards that Oversee Clinical Trials

- 21 CFR 312- Investigational New Drug Application 
- 21 CFR 314- Applications for FDA Approval to Market a new Drug

- 21 CFR 812- Investigational Device Exemptions

- 21 CFR 814- Premarket Approval of Medical Devices

These guidelines are self-explanatory and cover ethical, methodological and documentation aspects of clinical trials.

In research, a study subject's right, safety, well-being take precedence over the interests of science and society; and this can be achieved by following:

a) The GCP principles, which are consistent with principles of Declaration of Helsinki, and include the use of Informed Consent. Study staff respect subjects right by providing enough time and study related information so that the subject can voluntarily decide whether to participate in a trial or not.

b) By including provisions to maintain privacy and confidentiality of information regarding the subject, the subject is afforded respect and protection.

c) Subject's safety is ensured by having qualified (by education, experience and training) investigators and the study team to conduct the trial.

d) Subject's right, safety welfare is protected by Institutional Review Board (IRB) /Independent Ethics Committee that reviews and approves the study protocol, informed consent document, based on pre-decided parameters.

The FDA has authority of oversight over the IRB that must follow set standards of composition, function, operations procedures and records.

e) Study drug should be manufactured, handled, stored as per the approved protocol. Usage of the study drug as per approved protocol protects the subject safety and gives valid study results.

Implementation of quality assurance procedures in every aspect of clinical trial is important to assure valid, accurate, credible and acceptable data.

All clinical trial information should be recorded, handled, stored in a way to give accurate interpretation, verification and valid reports.

\section{Discussion}

ICH- GCP is required to be followed during studies conducted in the ICH region covering aspects such as design, conduct, performance, monitoring, auditing, recording, analysis and reporting of the clinical study. Regulators of countries in the ICH region would also accept data from studies conducted elsewhere, but according to ICH guidelines.

Data quality and integrity is important as entire clinical trial outcome depends on statistical analysis of the study data. Based on the clinical study report, a new drug may be cleared for marketing. Clinical trial results will be invalid if clinical study conduct and study data lack quality, integrity, ethics, sound scientific evidence, clarity and validity. If the data do not stand up to these standards, then an unsafe or inefficacious drug may be released to the market, while a safe and efficacious drug may be denied permission.

The ICH-GCP ensures protection of rights, safety and welfare of the human subjects, improves quality of data, decreases drug development cost to sponsors and user cost to the public and speeds up marketing of new drugs.

In last few decades clinical research has grown tremendously. There are multiple, complex studies in various phases, in various therapeutic areas going on across the globe. Harmonization ensures that the requirements are uniform all over and the data received from different sites can be pooled together to ensure that the studies have adequate power.

Quality conduct of clinical trial includes many aspects, such as ensuring:

1. Enrollment of eligible subjects on voluntarily basis, without coercion and by following informed consent guidelines (ICH- GCP), and FDA regulations (21 CFR part 50 subpart B) so as to protect right, safety, welfare of the study subject.

2. Accuracy of performed study procedures/event documentations per protocol.

3. Accuracy of collected study data supported by accurate, valid readable source documentation

(Example- medical record or original source document)

4. Timely review of study data and resolution of queries by study staff, which is verified by sponsor

5. Qualified/experienced principle investigator's oversight over the entire study conduct, which includes delegated experienced appropriately qualified, protocol/GCP trained study staff.

6. Validity of electronic system used in the study.

In a multicenter trial, which is going on in multiple countries, study result is compiled after analysis of study data collected from all study centers, all following the same common protocol. So it's important to have all study sites/ study personnel ICH-GCP trained and they should follow common ethical principles and procedures of clinical trial. If this happens, then only final study result will be credible without any bias.

\section{Advantages of ICH-GCP}

By providing uniform standards for planning, conducting, monitoring, auditing, recording and reporting clinical trials, ICH GCP ensures that clinical research conducted in different geographies come up to identical acceptable standards. It also ensures that the study subjects' right, safety, welfare, privacy and confidentiality are protected where the studies are conducted. The GCP guideline sends out a strong message to those working on drug development to desist from misconduct or fraud, since such incidence is quickly detected.

The guidelines provide the people an assurance that research subjects are treated with respect and dignity and that their well-being takes precedence over the interests of science and society, this ensures that people are motivated to participate in clinical trials, without whom no trial could ever be conducted.

The guideline guarantees that trials conducted at different centers follow the same quality standards and that the results can be extrapolated to all patients irrespective of race, gender etc. There is the additional benefit of mutual acceptance of trial data within ICH countries and even from centrist outside the ICH regions if the guidelines are followed. 
Citation: Wandile P, Ghooi R (2017) A Role of ICH- GCP in Clinical Trial Conduct. J Clin Res Bioeth 8: 1000297. doi: 10.4172/2155-9627.1000297

Page 5 of 5

These features hasten the drug development process, reduce the cost of drug development cost hence reducing the overall cost of healthcare.

\section{Conclusion}

The century that we live in, are perhaps the fastest developing. Inventions are taking place in the world at a pace never seen before. All fields, including the scientific are growing by leaps and bounds and the medical field is helping people live longer and healthier lives. A large part of this is due to the development of new drugs. The race to develop newer drugs or improve existing ones shows no sign of slowing.

In the past, clinical research was going on, but the pace was slow and development clumsy. There were many inconsistencies in health care delivery in different parts of the world, and these are now reducing. The world has not yet become a totally uniform society, but we are surely moving towards it.

The life of the human being has far more respect than it has ever had in the history. Uniform guidelines have ensured that the benefits of science are not restricted to one geography but are spread even across the world, as one geography moves ahead, so do all others.

Henry Ford said "If everyone is moving forward together, then success takes care of itself [9]".

\section{References}

1. Dixon JR (1988) The International Conference on Harmonization Good Clinical Practice guideline. Qual Assur 6: 65-74.

2. Dominguez-Urban I (1997) Harmonization in the regulation of pharmaceutical research and human rights: the need to think globally. Cornell Int Law J 30: 245-286.

3. http://www.fda.gov/downloads/Drugs/.../Guidances/ucm073122.pdf
4. http://www.fda.gov/downloads/Training/CDRHLearn/UCM176414.pdf

5. Gaw A (2014) Reality and revisionism: new evidence for Andrew C Ivy's claim to authorship of the Nuremberg Code. J R Soc Med 107: 138-143.

6. (1996) Nuremberg Doctor's Trial 1947. BMJ 313: 1445-1475.

7. Josefson D (1996) New US rules could overturn Nuremberg Code. BMJ 313: 1223.

8. Ghooi RB (2011) The Nuremberg Code A critique. Presp Clin Res 2: 72-76.

9. Kefauver-Harris amendments (2012) Revolutionized drug development. FDA consumer health information.

10. Riis P (1977) Letter from- Denmark. Planning of scientific-ethical committees. Brit Med J 2: 173-174.

11. Wolinsky H (2006) The battle of Helsinki: Two troublesome paragraphs in the Declaration of Helsinki are causing a furore over medical research ethics. ЕMBO Rep 7: 670-672.

12. Burgess LJ, Pretorius D (2012) FDA abandons the Declaration of Helsinki: The effect on the ethics of clinical trial conduct in South Africa and other developing countries. S Afr J BL 5: 87-90.

13. Gillon R (1985) "Primum non nocere" and the principle of nonmaleficence. Br Med J 291: 130-131.

14. ICH- History. http://www.ich.org/about/history.html

15. ICH- Process of Harmonization. http://www.ich.org/products/process-ofharmonisation.html

16. Grimm W (1998) Extension of the International Conference on Harmonization Tripartite Guideline for Stability Testing of New Drug Substances and Products to countries of climatic zones III and IV. Drug Dev Ind Pharm 24: 313-325.

17. Food and Drug Administration. Guidance for Industry E6 Good Clinical Practice: Consolidated Guidance. http://www.fda.gov/downloads/ Drugs/.../Guidances/ucm073122.pdf

18. Switula D (2000) Principles of good clinical practice (GCP) in clinical research. Sci Eng Ethics 6: 71-77.

19. http://www.accessdata.fda.gov/scripts/cdrh/cfdocs/cfcfr/cfrsearch.cfm 